\section{$\Omega$ MORTALITY IN CHILDREN WITH JUVENILE SYSTEMIC LUPUS ERYTHEMATOSUS IN AN ARGENTINE PEDIATRIC CENTRE.}

Cristina Battagliotti, Hospital de Niños Dr Orlando Alassia. ${ }^{11}$ Argentina, Santa Fe, Santa Fe, Argentina

Background: Juvenile systemic lupus erythematosus (JSLE) is a complex multisystemic autoimmune disease, often causing irreversible damage, reduced quality of life and life expectancy. Data on causes of death and time trends in infant lupus mortality are limited.

Objectives: The aim of this study was to determine mortality in a pediatric cohort

Methods: This retrospective study included patients with childhood onset lupus (fulfilling ACR 1997) who were diagnosed at the Paediatric centre in Santa Fe Argentine, Alassia Children Hospital from 1991 to 2018 and had 15 years of age at presentation.

Results: This study includes 54 JSLE children ( $F: M=12,5$ :1) with a mean age of onset lupus 12 years (range $4-15$ years). During a mean follow-up in our centre of 3 years (range $12 \mathrm{~m}-10$ years), 12 patients (24\%) was died; 5 during the follow-up in our centre and 7 in adult centre, 4 lost to follow-up.

The average age of death was 16 years (range 10-29 years) and the average since diagnose time was 17 months (range $3 \mathrm{~m}-15$ years).

The principal system involved was renal (100\%). 10 patients with diffuse proliferative lupus nephritis (WHO class IV), 2 membranous lupus nephritis (WHO class V), 5 patients $(41.6 \%)$ with hematological disorders (2 pancytopenia, 2 leukopenia, 1 Macrophage Activation Syndrome). 3 patients had thrombosis. 5 patients $(41.6 \%$ ) with neurological involvement (4 psychosis, 1 depression). One patient with liver dysfunction. There one death related to renal biopsy.

The principal cause of mortality was active disease and infection. Meningococcal meningitis, Klebsiella peritonitis, Pneumococcal cellulitis of the neck, peritonitis and 1 patient with disseminated tuberculosis.

11 patients (91,6\%) had received intravenous steroids as pulses and Cyclophosphamide pulse therapy, azathioprine or mycophenolate mofetil, hydroxychloroquine immunoglobulins. In some patients peritoned dialysis and ventilation. 8 patients $(66 \%)$ with bad socioeconomic environment. Four patients $(33 \%)$ with poor adherence to treatment.

Conclusion: In this single-center study, childhood onset lupus was associated with a mortality of $24 \%$. With higher frequency of aggressive renal disease and higher requirement for steroids and immunosuppressive drugs. Pediatric care should consider transition to adult's centers and ensure continuity of treatment.

\section{REFERENCE}

[1] Childhood systemic lupus erythematosus in Latin America. The GLADEL experience in 230 children. February 2008 Lupus 17(6):596-604 DOI: $10.1177 / 0961203307088006$

Acknowledgement: Hospital de Niños Dr Orlando Alassia

Disclosure of Interests: None declared

DOI: 10.1136/annrheumdis-2019-eular.8270

\section{SAT0187 \\ TRENDS IN EMPLOYMENT AND HOSPITALIZATION IN PATIENTS WITH SJÖGREN'S SYNDROME 1993-2016: RESULTS FROM THE GERMAN NATIONAL DATABASE}

Johanna Callhoff ${ }^{1}$, Katja Thiele ${ }^{1}$, Thomas Dörner ${ }^{2}$, Angela Zink ${ }^{1,2}$, Jörg Henes ${ }^{3}$, Jutta Richter ${ }^{4}$, Katinka Albrechtt. ${ }^{1}$ German Rheumatism Research Centre, Epidemiology, Berlin, Germany, ${ }^{2}$ Charité University Medicine, Berlin, Germany; ${ }^{3}$ University Hospital Tübingen, Rheumatology, Tübingen, Germany; ${ }^{4}$ HeinrichHeine-University, Department of Rheumatology and Hiller Research Unit, Düsseldorf, Germany

Background: During the last 20 years, employment rates increased substantially in the German population and also in patients with arthritis [1] Whether patients with primary Sjögren's syndrome (pSS) also show this trend is less clear in the absence of new treatment options.

Objectives: To assess trends in treatment and outcomes in patients pSS, focusing on employment, hospitalization and medical treatment in the past two decades.

Methods: From 1996 to 2016, 300 patients with pSS were documented annually in the National Database of the German Collaborative Arthritis Centres. Data on treatment, physician assessment of disease activity, patient-reported outcomes, hospitalization and employment were collected and compared to patients with rheumatoid arthritis (RA), matched 1:1 for age, sex and disease duration for each calendar year.
Results: Patients with pSS ( $>90 \%$ female, age $\sim 44$ years at disease onset, disease duration $\sim 10$ years) were more frequently assessed to be in low disease activity in 2016 (93\%) than in 1996 (62\%, p<0.01). Treatment with antimalarials increased $(31 \%$ to $50 \%, \mathrm{p}<0.01)$ and less patients were on glucocorticoids $(50 \%$ to $34 \%, p<0.01)$; Less than $5 \%$ were treated with biologics in 2016. The percentage of employed patients $(<65$ years) increased by 21 percentage points ( $43 \%$ to $64 \%, p<0.001)$, exceeding the increase observed for RA patients $(+15$ percentage points) In 2016, significantly less patients compared to 1996 were on early retirement $(22 \%$ and $10 \%, \mathrm{p}=0.01$ ), hospitalized/year ( 13 and $7 \%, \mathrm{p}=0.08$ ) or on temporary sick leave $(27 \%$ compared to $39 \%, p=0.09)$. This trend is comparable to RA patients.
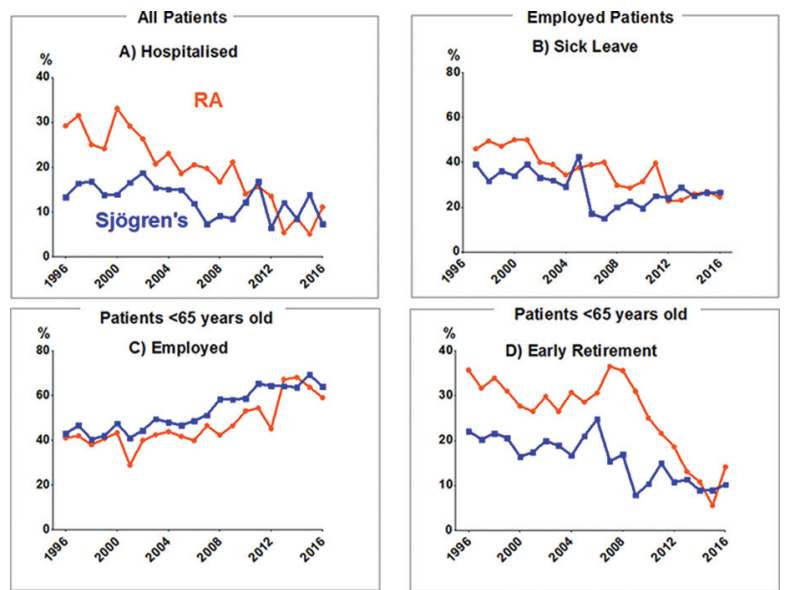

Conclusion: Overall, similar trends were observed for RA and pSS cohorts despite minor changes in pSS therapy. Work participation has improved significantly over two decades in pSS. A greater perception of pSS without systemic manifestations may have caused a shift towards less severely affected patient cohorts today.

\section{REFERENCE}

[1] Mau W, Listing J, Huscher D, Zeidler H, Zink A. Employment across chronic inflammatory rheumatic diseases and comparison with the general population. J Rheumatol. 2005;32(4):721-8

Acknowledgement: The database is funded by unconditional grants from the German Collaborative Arthritis Centres and from a consortium of 9 pharmaceutical companies to the German Academy for Continuing Medical Education in Rheumatology.

Disclosure of Interests: Johanna Callhoff: None declared, Katja Thiele: None declared, Thomas Dörner Grant/research support from: Eli Lilly, Janssen, Roche, UCB Pharma, Consultant for: Eli Lilly, Janssen, Roche, UCB Pharma, Speakers bureau: Eli Lilly, Janssen, Angela Zink Speakers bureau: Speakers fees from AbbVie, Janssen, Pfizer, Roche, Sanofi, Jörg Henes: None declared, Jutta Richter Grant/research support from: GlaxoSmithKline and UCB Pharma for performing the LuLa-study., Katinka Albrecht: None declared

DOI: 10.1136/annrheumdis-2019-eular.1183

\section{SAT0188 WHOLE BLOOD VERSUS SERUM HYDROXYCHLOROQUINE LEVELS FOR DRUG MONITORING OF PATIENTS WITH SYSTEMIC LUPUS ERYTHEMATOSUS: PRELIMINARY RESULTS OF A PHARMACOLOGICAL STUDY}

Benoit Blanchet ${ }^{1}$, Moez Jallouli ${ }^{1}$, Marie Allard ${ }^{1}$, Pascale Ghillani Dalbin², Lionel Galicier ${ }^{3}$, Véronique Le Guern ${ }^{1}$, Noel Zahr ${ }^{2}$, Claire Goulvestre ${ }^{1}$,

Zahir Amoura ${ }^{2}$, Jean-Charles Piette ${ }^{2}$, Noemie Jourde-Chiche ${ }^{4}$, Nathalie CostedoatChalumeau ${ }^{1}$, Group PLUS. ${ }^{1}$ Cochin Hospital, Paris, France; ${ }^{2}$ Pitié Salpêtrière Hospital, Paris, France; ${ }^{3}$ Saint Louis Hospital, Paris, France; ${ }^{4}$ Aix-Marseille Univ, C2VN, INSERM, INRA, AP-HM Centre de Néphrologie, Marseille, France

Background: In order to assess the pharmacokinetic/pharmacodynamic relationship of hydroxychloroquine (HCQ) in patients with systemic lupus erythematosus (SLE), HCQ levels have been measured in whole blood as well as in serum but both methods have never been compared. In addition, cut offs for non-adherence (classically $200 \mathrm{ng} / \mathrm{ml}$ but also 100 $\mathrm{ng} / \mathrm{mL}$ ) have been established only in whole blood. 
Objectives: The aims of this study were (1) to compare these two pharmacological approaches, and (2) since it would be very interesting to retrospectively assess severe non-adherence in clinical trials or in large cohort of patients in which only serum samples are usually available, to determine if serum HCQ level cut offs could be established for identification of severe non-adherent patients.

Methods: The HCQ and desethylchloroquine (DCQ) levels were measured in serum and whole blood from 573 SLE patients. The risk factors for active SLE (SLEDAI score >4) were identified using multiple logistic regression. $\mathrm{HCQ}$ serum level was also measured in 51 non-adherent patients (whole blood $\mathrm{HCQ}$ level $<200 \mathrm{ng} / \mathrm{mL}$ ).

Results: The mean HCQ and DCQ levels were $916 \pm 449$ and $116 \pm$ $55 \mathrm{ng} / \mathrm{mL}$ in whole blood, respectively; and $469 \pm 223$ and $63 \pm 31 \mathrm{ng} /$ $\mathrm{mL}$ in serum, respectively. The mean ratio of serum/whole blood level for $\mathrm{HCQ}$ and $\mathrm{DCQ}$ were $0.53 \pm 0.15$ and $0.57 \pm 0.21$, respectively. $\mathrm{A}$ strong positive correlation was found between serum and whole blood levels of $\mathrm{HCQ}$ (rho=0.837 [Cl95\% 0.810-0.860], $\mathrm{p}<0.0001$ ), and $\mathrm{DCQ}$ (rho=0.771 [Cl95\% 0.736-0.802], $\mathrm{p}<0.0001)$. In the multivariate analysis, only corticosteroids $(p=0.044)$, immunosuppressant $(p=0.027), \mathrm{HCQ}$ whole blood level $(p=0.023)$ and hemoglobin $(p=0.009)$ were identified as an independent risk factor of active SLE but serum HCQ level was not. Given the mean ratio of serum/whole blood level for $\mathrm{HCQ}$ was 0.53 , we extrapolated that serum $\mathrm{HCQ}$ level cut offs of 106 and $53 \mathrm{ng} / \mathrm{mL}$ would correspond to the previously used cut-off of 200 and $100 \mathrm{ng} / \mathrm{mL}$ of $\mathrm{HCQ}$ in whole blood.

Using $\mathrm{HCQ}$ serum level cut off of $106 \mathrm{ng} / \mathrm{mL}, 43$ of 51 patients $(84 \%)$ with blood $\mathrm{HCQ}$ levels $<200 \mathrm{ng} / \mathrm{mL}$ would also have been considered as non-adherent. The positive and negative predictive value of $\mathrm{HCQ}$ serum level < $106 \mathrm{ng} / \mathrm{ml}$ to detect non-adherence were $96.6 \%$ and $63.6 \%$, respectively.

Of these 51 patients, 25 patients (49\%) exhibited $\mathrm{HCQ}$ whole blood concentration below $100 \mathrm{ng} / \mathrm{mL}$. Using $\mathrm{HCQ}$ serum level cut off of $53 \mathrm{ng} /$ $\mathrm{mL}, 23$ of 25 patients (92\%) with $\mathrm{HCQ}$ whole blood level $<100 \mathrm{ng} / \mathrm{mL}$, would also have been considered as non-adherent. The positive and negative predictive value of $\mathrm{HCQ}$ serum level $<53 \mathrm{ng} / \mathrm{ml}$ to detect nonadherence were $82.1 \%$ and $90.9 \%$.

Conclusion: Our data support the use of whole blood rather than serum as the matrix for drug monitoring of HCQ levels in SLE patients. However, when whole blood is not available, our results support the use of $\mathrm{HCQ}$ serum level to assess non-adherence with a cut off of $106 \mathrm{ng} / \mathrm{mL}$ corresponding to $200 \mathrm{ng} / \mathrm{ml}$ in whole blood.

Disclosure of Interests: None declared

DOI: 10.1136/annrheumdis-2019-eular.4215

\section{SAT0189 A NOVEL DEVICE FOR RAPID MINOR SALIVARY GLAND BIOPSY IN SUSPECTED SJÖGREN'S SYNDROME}

Alexandre Dumusc $^{1}$, Bettina Bannert ${ }^{2}$, Diana Dan ${ }^{1}$, Thomas Huegle ${ }^{1} .{ }^{1}$ University Hospital Lausanne (CHUV), Rheumatology, Lausanne, Switzerland; ${ }^{2}$ Basel University Hospital (USB), Rheumatology, Basel, Switzerland

Background: Labial minor salivary glands (MSG) biopsies are a frequently performed diagnostic procedure for Sjögren's syndrome. MSG biopsy usually requires additional assistance e.g. to provide lower lip protrusion and to apply pressure on the lip during the procedure. The latter is done to foster protrusion of salivary glands and therefore to reduce the incision depth and potential nerve damage. We recently developed a new singleuse device for MSG by 3D-printing in form of a lip clamp with a stamp on the lower arm to reduce invasiveness and to facilitate the intervention.

Objectives: To assess practicability and to describe the technical application of this device for MSG biopsies in the rheumatology department

Methods: Retrospective study of MSG biopsies performed with the use of a dedicated lip clamp. For each MSG biopsy, we recorded if any assistance was needed during the procedure, occurrence of any complication, if salivary glands were obtained and if the quality of the salivary gland tissue sample was adequate for histologic analysis.

Results: 12 MSG biopsies performed on 12 patients with suspected Sjögren's syndrome were included in the study. MSG biopsies were performed between August 2018 and January 2019. Using the lip clamp, the physician was able to perform all the biopsies without assistance. No complication occurred with a follow-up of 1 week for each patient. Salivary glands tissue samples were obtained in $11 / 12$ of the patients using 1 incision. For 1 patient, a second incision had to be performed on the contralateral side of the lip during the same intervention because no glands were found on the first attempt. Quality of all the samples was adequate for histologic analysis. We did not observe an increase in procedure time compared to routine MSG biopsy procedure.

Conclusion: The use of a single-use lip clamp facilitates MSG biopsy without needing assistance and potentially reduces invasiveness and collateral damage. Quality of the tissue samples obtained was adequate for histological analysis.
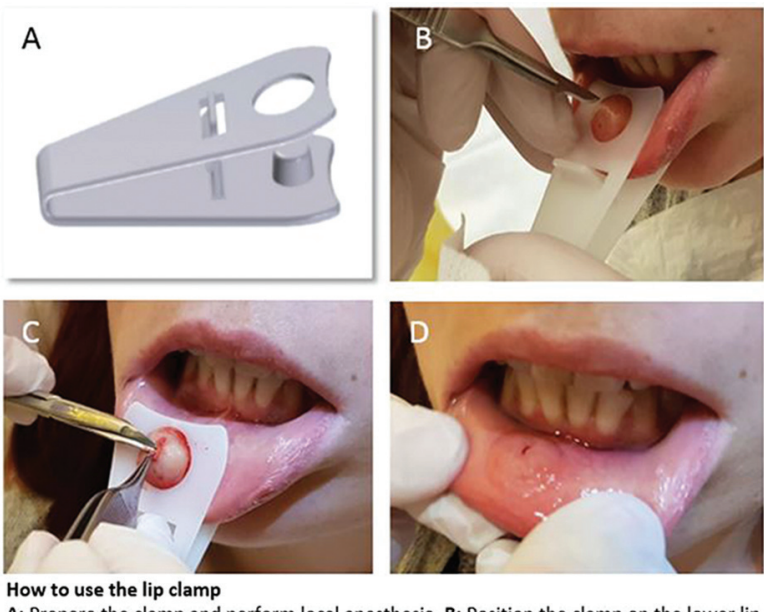

(hesthesia. B: Position the clamp on the lower lip with the recess of the clamp facing up, at $0.5-1 \mathrm{~cm}$ off the midline. C: Perform a $5 \mathrm{~mm}$ vertical superficial incision. Due to the pressure from below, a minor salivary gland vertical superficial incision. Due to the pressure from below, a minor salivary gland
appears. Seize it with forceps and cut it with scissors or scalpel. D: Open and remove the clamp. No stiches are required.

Disclosure of Interests: Alexandre Dumusc: None declared, Bettina Bannert: None declared, Diana Dan: None declared, Thomas Huegle Grant research support from: AbbVie, Lilly, Novartis and Pfizer, Speakers bureau: AbbVie, Lilly, Novartis and Pfizer

DOI: 10.1136/annrheumdis-2019-eular.5974

\section{SAT0190 LYMPHOMA IN PRIMARY SJÖGREN'S SYNDROME: A RETROSPECTIVE CLINICAL STUDY WITH PATIENTS FROM THE UPA (UDINE, PISA, ATHENS) GROUP}

Andreas Goules ${ }^{1}$, Chiara Baldini ${ }^{2}$, Saviana Gandolfo ${ }^{3}$, Aristea Papageorgiou ${ }^{4}$, Dimitris Ziogas ${ }^{4}$, Francesco Ferro ${ }^{2}$, Valentina Donati ${ }^{2}$, Cathryn Scott ${ }^{3}$, Salvatore De Vita ${ }^{3}$, Michael Voulgarelis ${ }^{4}$, Athanasios Tzioufas ${ }^{5} .{ }^{1}$ Athens School of Medicine, National and Kapodistrian University of Athens, Athens, Greece., Pathophysiology, Athens, Greece; ${ }^{2}$ University of Pisa, Rheumatology Unit, Department of Clinical and Experimental Medicine, Pisa, Italy; ${ }^{3}$ University of Udine, Department of Medical and Biological Sciences, Rheumatology Clinic, Udine, Italy; ${ }^{4}$ Athens School of Medicine, National and Kapodistrian University of Athens, Athens, Greece., Pathophysiology, Athens, Greece; ${ }^{5}$ Athens School of Medicine, National and Kapodistrian University of Athens, Athens, Greece., Pahtophysiology, Athens, Greece

Background: Lymphoma development in Sjögren's syndrome has significant impact on morbidity and mortality of the disease.

Objectives: To describe and compare clinical, serological and histological features of patients with primary Sjögren's syndrome (pSS) associated non-Hodgkin's lymphomas (NHLs) in 3 cohorts, from Greece (1) and Italy (2).

Methods: 140 consecutive pSS patients with NHLs who fulfilled the 2002 AECG criteria for Sjögren's were included in the study. Patients were recruited from 3 centers (Udine, Pisa, Athens, cohort named UPA), two from Italy (Udine and Pisa University Hospitals with a total of 63 cases, named cohort UP) and one from Greece (University of Athens with 77 cases, named cohort $\mathrm{A}$ ). Age at $\mathrm{NHL}$ onset, $\mathrm{NHL}$ subtype and distribution, SS disease duration until lymphoma development, serum anti-SSA SSB antibodies and rheumatoid factor, history of salivary gland enlargement, cryoglobulinemia with or without vasculitis and $\mathrm{C} 4$ hypocomplementemia before the onset of $\mathrm{NHL}$ were recorded and compared.

Results: The Greek cohort (A) had a median age at lymphoma diagnosis 58 years (range: $28-90$ years) and the (UP) cohort a median age a diagnosis of lymphoma 55.6 years (range: $25-77$ years). The median time from SS to lymphoma diagnosis was 65.8 months (range: 0-456 months) vs 48 months (range: 0-276 months) for (A) and (UP) cohort respectively. The commonest histologic subtypes such as, MALT (mucosa associated lymphoid tissue), diffuse large B cell (DLBC) and nodal or splenic marginal zone (MZ) lymphomas were observed in similar frequencies between 